\title{
Immigration, integration and ghetto formation
}

\author{
Hildegard Meyer-Ortmanns \\ School of Engineering and Science \\ International University Bremen \\ P.O.Box 750561 \\ D-28725 Bremen, Germany \\ e-mail: h.ortmanns@iu-bremen.de
}

June 22, 2021

\begin{abstract}
We study ghetto formation in a population with natives and immigrants in the framework of the two-dimensional Ising-model with Kawasaki-exchange dynamics. It is the phase structure of the Ising model, the integration speed and the immigration rate which determine whether ghetto formation between natives and immigrants can be avoided or not. Our simulations are performed in and out-of equilibrium.
\end{abstract}

Keywords: sociophysics, ghetto formation, Monte Carlo, Ising-Kawasaki dynamics

\section{Introduction}

Sociophysics as a branch of interdisciplinary research has recently attracted more attention (for a review see [1] and for the background [2]). Models and tools of statistical physics turned out to be successful in reproducing and predicting features of traffic [3], migrations [4], and opinion formation in social groups [5].

In this paper we study the conditions for ghetto formation [6] in a population with natives and immigrants by using Kawasaki-exchange dynamics [7] in a twodimensional Ising model. To provide the background from physics we summarize some well known features about the phase structure of the Ising model in section 2 . An outline of our measurements that are supposed to imitate realistic situations is given in section 3 . In section 4 we present the results about ghetto formation. The simulations are parametrized by the immigration rate and the integration efforts. Section 5 contains the summary and conclusions. 


\section{Background from physics}

We study the Ising model in two dimensions with fixed magnetization. So-called spins $\sigma$ are associated with the sites of the lattice (here assumed to be rectangular) taking values of +1 or -1 . These values could stand for the orientation of the magnetic moment in a ferromagnet, or for the presence or absence of an atom in a liquid, or labels for two different metals. In our application they stand for an immigrant $(+1)$ and a native $(-1)$ in a certain human population. The energy derives from the interaction between neighboring spins. The energy per pair of nearest neighbors $x$ and $y$ is given by $-J \cdot \sigma(x) \sigma(y)$, where $J$ is the spin-spin interaction. Thus the interaction energy is $-J$ if the spins are aligned and $+J$ if they are different. When the temperature is high as compared to $J$, the spins are disordered. However, below a critical temperature $T_{c}$, at zero external field, there is a spontaneous magnetization quantified by the non-vanishing order parameter $m=\langle\sigma\rangle$, the weighted average over the spin. Spins of equal sign like to align and order into a state of broken symmetry. For fixed $T<T_{c}$, at zero magnetic field $h$, there are coexisting states between the two different ordered phases with order parameters $\pm m$, respectively. A plot of $T( \pm m)$ for $T \leq T_{c}$ results in the magnetization curve. We choose the m-axis as abscissa and the T-axis as ordinate. Values $(m, T)$ below this curve are forbidden parameters in equilibrium in the sense that they lead to unstable or metastable configurations, because they correspond to values of the magnetization, which would not form spontaneously.

If the magnetization is kept fixed, however, as in the Kawasaki dynamics, $(m, T)$ values below the curve lead to phase separation in the form of infinite-range clusters. In a finite volume, these clusters have a radius proportional to the linear size of the system. We call these infinite-range clusters ghettos. Like ghettos these clusters are self-supporting in the sense that spins (immigrants) can move over a distance of the whole lattice (city, country, their own "world") without meeting spins of opposite sign (natives, respectively). Infinite-range clusters should be distinguished from finite-range correlation volumes with a radius $r \ll L$. Such correlated regions correspond to water droplets in the fog with varying size, which stay, however, finite, when the system size goes to infinity. Finite-range clusters will be found as equilibrium configurations for $(m, T)$ values above the magnetization curve.

One way of investigating the phase structure of this model is the Monte Carlo method. The "classical" way is Glauber dynamics, realized, for example, in the Metropolis algorithm. In each step of this algorithm, one proposes to flip a single spin with an acceptance probability such that each state occurs with the correct probability. In this approach the order parameter fluctuates. In the Kawasaki-exchange dynamics, nearest-neighbor spins are exchanged under heat-bath dynamics, i.e. with probability $1 /\left(1+\exp \left(\Delta E / k_{B} T\right)\right)$, where $\Delta E$ is the energy change under the spin exchange. This way the order parameter, here the total magnetization, is conserved during the evolution of the system.

In case of the population with natives and immigrants the parameter $k_{B} T$ plays 
the role of the so-called social temperature. The social temperature accounts for the possibility of learning, assimilation and integration of immigrants whoever takes the initiative. For simplicity, the population size (total number of spins) is kept constant during the evolution. We consider the concentration $c$ of immigrants, $c \in[0,1]$, rather than the magnetization. Both quantities are related via $c=(m+1) / 2$, $m \in[-1,+1]$. The magnetization curve will then be replaced by the coexistence curve. (The more generic name shall indicate that along and below this curve different phases may coexist.)

In this dynamics the exchange of spins proceeds only locally in space, while in reality immigrants will make non-local moves from one place to another. We do not expect that our qualitative results would change if we skip this simplification and allow also for exchange with next-to-next nearest neighbors, for example, or other local moves. The classical Schelling model is roughly a low-temperature simulation of this Kawasaki model but also allowing for many empty sites [6].

\section{Measurements}

We start with a population of $L_{1} \times L_{2}$ spins on a square lattice, initially all chosen to -1 (natives). In a first sweep we introduce immigrants by flipping randomly chosen -1 -spins with probability $c$. The initial population then consists of $(1-$ c) $\cdot L_{1} \cdot L_{2}$ natives and $c \cdot L_{1} \cdot L_{2}$ immigrants, randomly distributed among the natives. The evolution of the system under an exchange of natives and immigrants with probability $1 /\left(1+\exp \left(\Delta E / k_{B} T\right)\right)$ is then followed over $N$ time steps, i.e. over on average $N$ updates of every spin. Observables are measured on the resulting configuration. In particular we take a snapshot of the configuration as they are displayed in the various figures below. We then repeat the set of $N$ measurements a number of $k$-Monte Carlo step times. Such repetitions serve to improve the statistics if $N$ was chosen large enough as to give an equilibrium result. For example, on a lattice of linear size $L$ it takes about $N \propto L^{3}$ simulations to produce a ghetto with radius $R \sim L$ for a point $(c, T)$ below the coexistence curve. If $N \ll L^{3}$, the kmany repetitions of the first $N$ sweeps lead to different snapshots, and values of the observables differ from the first one by more than statistical fluctuations. This is just a manifestation of the out-of equilibrium simulations.

In realistic immigration and integration procedures the number of immigrants grows for a while up to a certain saturation, and integration measures are supported with varying intensity. During our runs we therefore vary the social temperature with speed $\Delta T / \Delta t$ as well as the concentration of immigrants with a rate $\Delta c / \Delta t$, $\Delta t$ being a time interval in Monte Carlo steps per spin. Alternatively the final outcome can be parametrized by $\Delta T / \Delta c$, the slope of the straight line connecting the different measuring points in the $(c, T)$-diagram. For each of the selected $(c, T)$ points we perform $k$-times $N$ evolution steps, where $N$ varies not only with $(c, T)$, but also for fixed $(c, T)$ to study the in-and off-equilibrium effects. 


\section{Results}

We choose a square lattice of size $79 \times 100$, where the (unusual) choice of 79 is just to facilitate the print of the snapshots of the spin configurations (the $\star$ stand for immigrants, the blanks for natives). We choose periodic boundary conditions. In Fig.1-4 we display the results of four runs.

- Ghetto-dissolution.

The first run is at constant immigration concentration of $20 \%$ starting at a temperature $T / T_{c}=0.8$ below the concentration curve with $N=10^{7}$ sweeps, leading to a ghetto of immigrants (Fig.1a). At constant concentration the integration effort $(T)$ is then instantaneously increased to $T / T_{c}=1.2$ above the $(c, T)$-curve. (Such a quench in temperature may reflect a political decision to support the early education in the native language and the like.) The Figs.1b-c (after 100 (1b) and 10000 (1c) iterations) then show that the ghetto does not dissolve instantaneously. The time it takes until all remnants of the ghetto have disappeared after $10^{6}$ iterations (as shown in Fig.1d) is comparable to the time as it took to create it (between $10^{6}$ and $10^{7}$ iterations). This symmetry is due to the Kawasaki-exchange dynamics and the location in phase space.

- Ghetto formation.

Here all $(c, T)$ points are below the concentration curve. For equilibrium simulations we therefore expect ghetto formation. We start with an initial concentration of $15 \%$ at $T / T_{c}=0.8$, simulate little integration efforts $(\Delta T / \Delta t=0.025 / 600)$, although the immigration rate $\Delta c / \Delta t=0.05 / 600$ is high, corresponding to a small slope $\Delta T / \Delta c=0.025 / 0.05$ in the $(c-T)$ diagram. Fig.s $2 \mathrm{a}$ and $2 \mathrm{~b}$ show populations with $\left(c, T / T_{c}\right)=(0.15,0.8)$ and $(0.25,0.85)$, respectively, after 600 iterations both, not yet equilibrated, while Fig.s $2 c$ and d show how a ghetto is formed for $\left(c, T / T_{c}\right)=(0.35,0.9)$ after $N=10^{3}(2 \mathrm{c})$ and $N=10^{7}(2 \mathrm{~d})$ iterations. The equilibrium state (2d) shows a ghetto. Opposite to the course in 1) it takes time to form a ghetto ( $10^{7}$ time steps), but it is the unavoidable fate if $\Delta T / \Delta t$ is too small and $\Delta c / \Delta t$ is too large. In other words, politicians in favor of small integration efforts in spite of a high immigration rate will be late and encourage ghetto formation.

- No ghetto formation at all.

These results for $\left(c, T / T_{c}\right)$ points $(0.05,0.8)$ (Fig.3a), (0.10,0.95) (Fig.3b), $(0.15,1.1)($ Fig.3c) and $(0.20,1.25)$ (Fig.3d), all above the $(c-T)$-curve, after $10^{7}$ iterations certainly in equilibrium, shall illustrate that there are situations where ghettos are avoided. However small the integration efforts are while the immigration slowly increases, no ghettos will form. This is what one may have naively expected to hold in other regions of the phase diagram as well. It is the well understood phase structure of the Ising model simulated with Kawasaki dynamics that tells us for which $\left(c, T / T_{c}\right)$ values the naive expectation fails and for which it holds. 
- No ghetto formation as an off-equilibrium effect.

Here we start with an initial concentration of $15 \%$ immigrants at a temperature of $T / T_{c}=0.8$ (Fig.4a) after $N=600$ iterations). These are $\left(c, T / T_{c}\right)$ parameters for which the immigrants would form a ghetto if we wait until the evolution has reached its equilibrium state. However, in our simulations we do not wait that long but increase $T$ while $c$ increases as well $(0.25,0.85)$ (Fig.4b) and $(0.35,0.90)$ (Fig.4c)), both after 600 iterations. Next we limit the immigration to $35 \%$ and further increase the integration measures to $T / T_{c}=1.2$ so that we reach the area of phase space above the $(c-T)$-curve where no ghettos will form. There we wait $10^{7}$ iterations until the equilibrium situation has been reached, see the snapshot in Fig.4d. The Fig.'s 4 demonstrate that no ghettos will form whatever our waiting time. The little clusters of immigrants as they are still seen in Fig.4d correspond to "droplets in the fog" rather than to an "ocean below the atmosphere" which would be called a ghetto in our context. In other words: With an appropriate choice of $\Delta T / \Delta t$ and $\Delta T / \Delta c$ politicians can avoid ghetto formation, even if the initial conditions do not look promising on a long-time scale (equilibrium scale).

\section{Summary and Conclusions}

A general remark is in order about the meaning and value of numbers produced in our simulations in view of applications to social systems. Certainly none of the parameter values like the lattice size $L_{1} \times L_{2}$, the social temperature, the time measured in units of Monte Carlo iterations should be taken literally. Obviously a realistic typical population size in which one would study the phenomenon of ghetto formation may exceed our choice of $79 \times 100$ "spins". Time would be measured in units of months and years. The social temperature is an effective parameter. It provides one but certainly not the only possibility to take integration measures into account. However, what should survive simulations with more realistic parameters will be the qualitative features summarized in section 4. An important common feature between social systems and our simulations is the state of being out-of-equilibrium, at least for intermediate periods. It was this feature that gave the chance of avoiding ghettos even if the initial conditions look discouraging under equilibrium conditions. In summary we have shown that depending on the integration efforts and the immigration rate politicians can be both late or in time for avoiding ghetto formation.

\section{Acknowlegdment}

It is a pleasure to thank Wolfgang Weidlich (Stuttgart) and Dietrich Stauffer (Köln) for stimulating discussions during the conference on Sociophysics (June 6-9, 2002) at the Center for Interdisciplinary Research (ZiF)in Bielefeld. 


\section{References}

[1] D. Stauffer, Journal of Arificial Societies and Social Simulation, vol.5 issue 1 paper 4 (2002) (jasss.soc.surrey.ac.uk); S. Moss de Oliveira, P. M. C. de Oliveira, and D. Stauffer, Evolution, Money, War and Computers (Teubner, StuttgartLeipzig 1999).

[2] W. Weidlich, Sociodynamics: A Systematic Approach to Mathematical Modelling in the Social Sciences. Harwood Academic Publishers, 2000.

[3] D. Helbing, Phys. Rev. E 55, 3735 (1997).

[4] W. Weidlich, G. Haag, Concepts and Models of Quantitatively Sociology, Springer, Berlin, 1983; W. Weidlich, Phys.Rep.204, 1 (1991)

[5] K. Kacperski and J. A. Holyst, Physica A269, 511 (1999); ibid 287, 631 (2000); F. Schweitzer and J. A. Holyst, Eur.J.Phys.B15, 723 (2000).

[6] T. C. Schelling, J.Mathematical Sociology, 1, 143 (1971).

[7] P. Tamayo and W. Klein, Phys.Rev.Lett.63, 2757 (1989); 66, 2049 (1991); A. J. Bray, ibid.66,2048 (1991). 


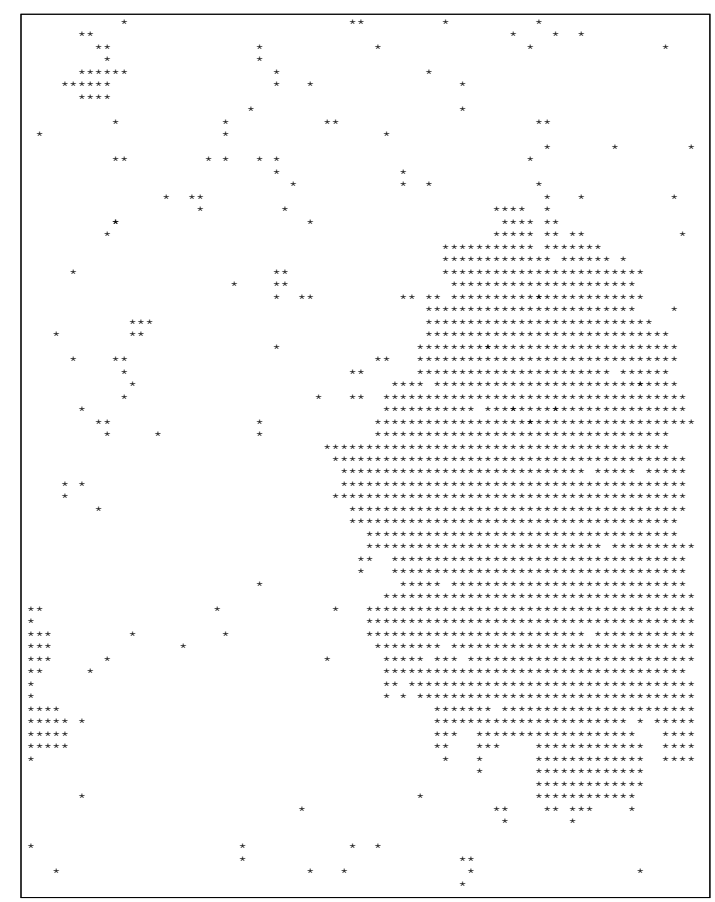

Fig.1a: $c=0.2, T / T_{c}=0.8, N=10^{7}$

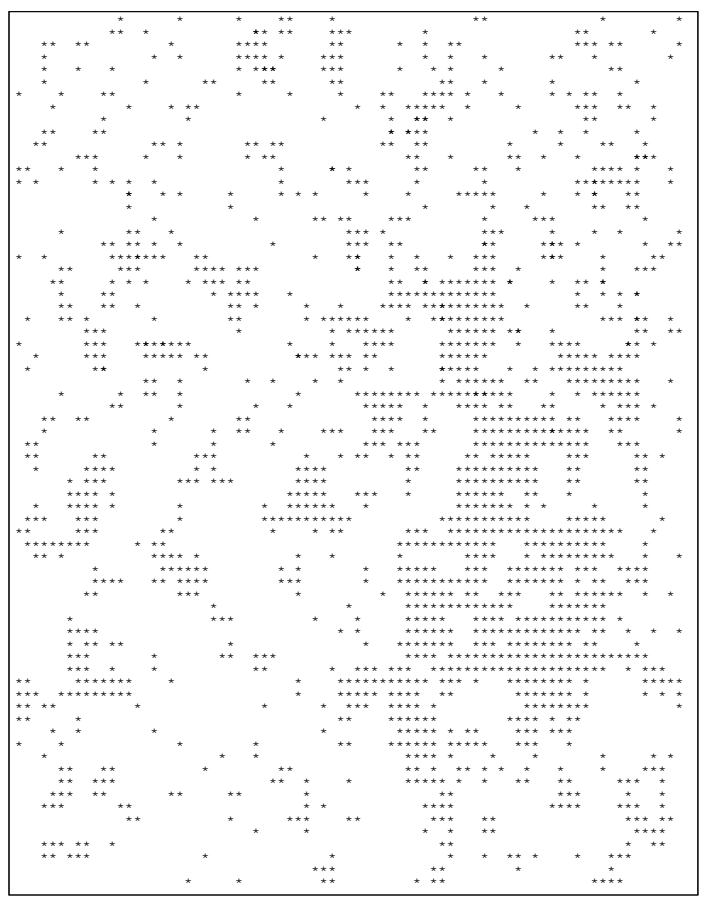

Fig.1c: $c=0.2, T / T_{c}=1.2, N=10^{4}$.

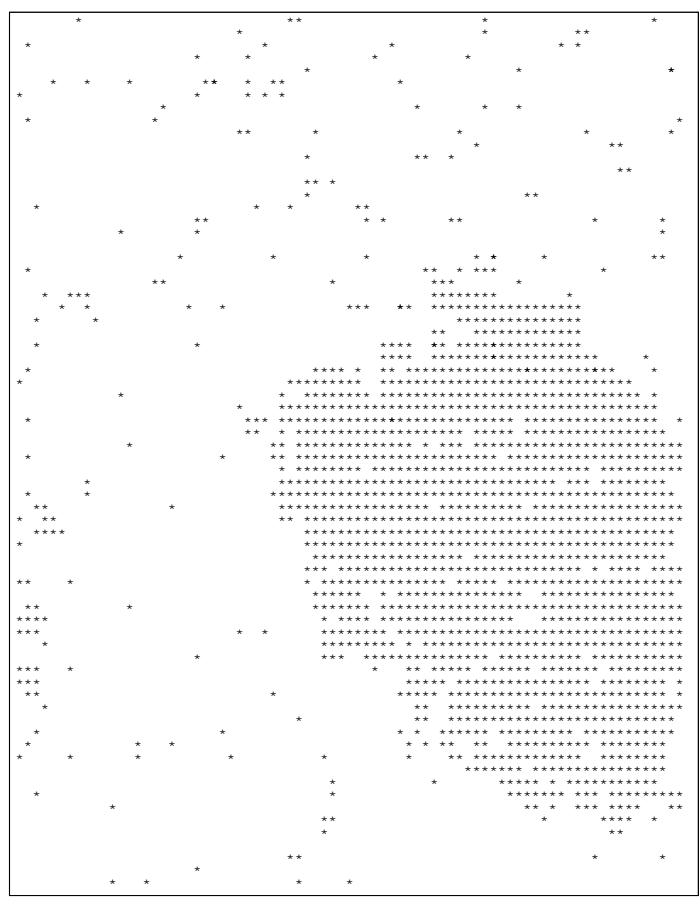

Fig.1b: $c=0.2, T / T_{c}=1.2, N=10^{2}$

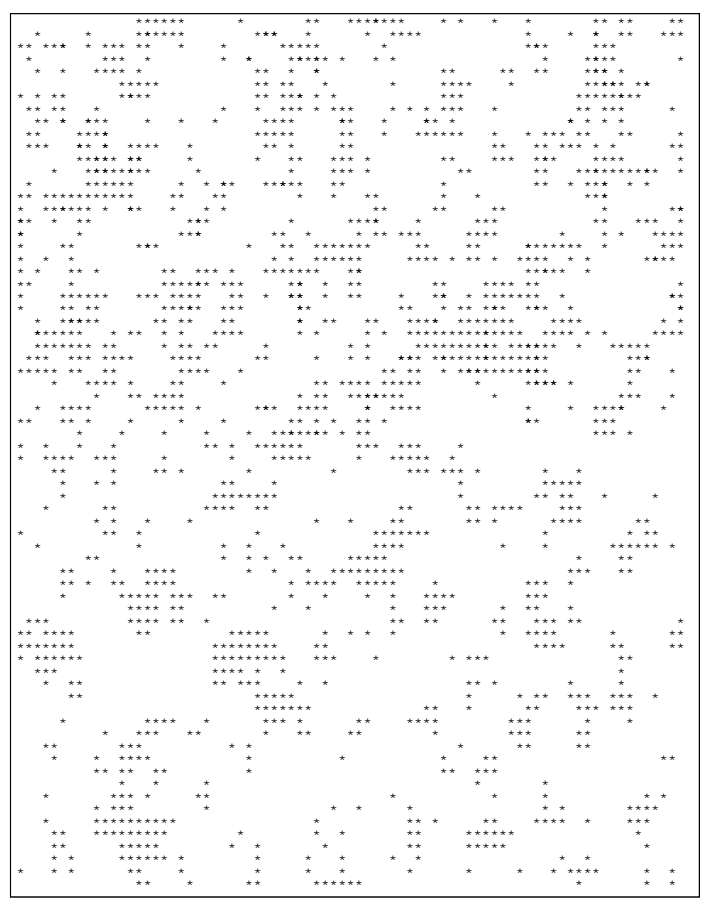

Fig.1d: $c=0.2, T / T_{c}=1.2, N=10^{6}$

Figure 1: Dissolution of a ghetto of immigrants $(\star)$ in a population of natives (blanks). 


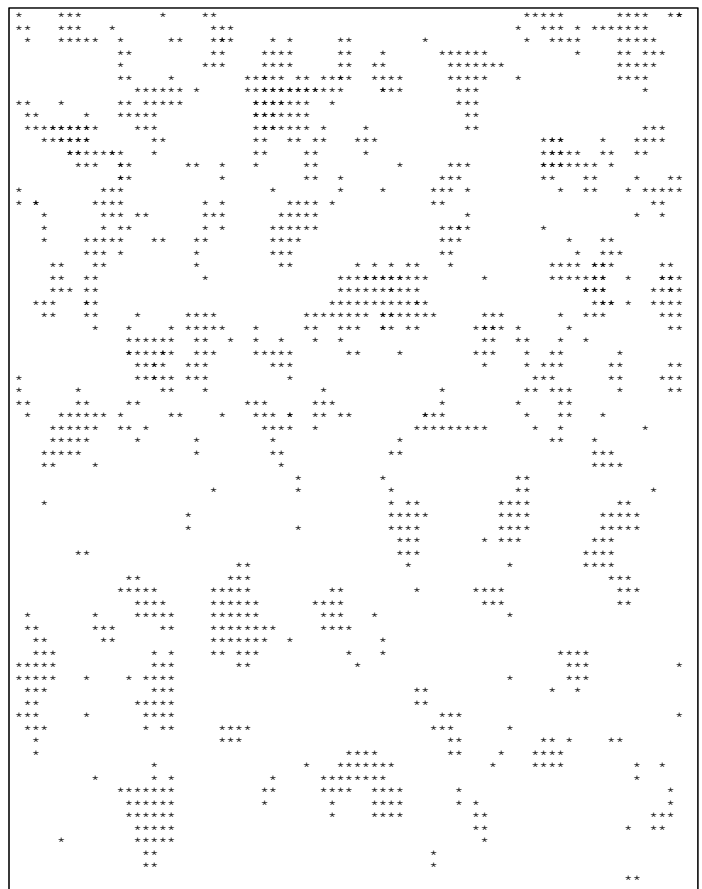

Fig.2a: $c=0.15, T / T_{c}=0.8, N=600$

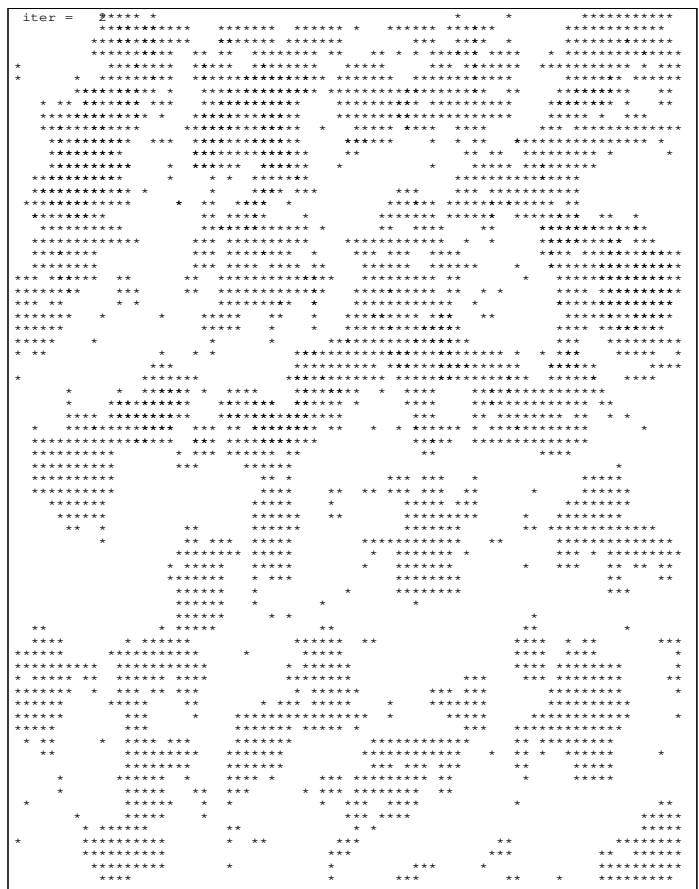

Fig.2c: $c=0.35, T / T_{c}=0.9, N=10^{3}$

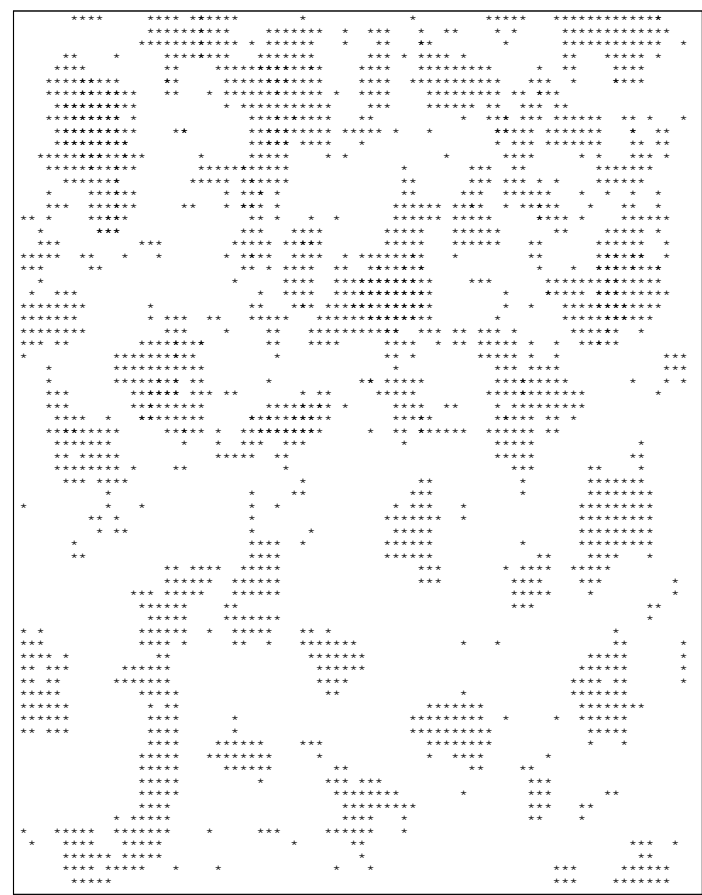

Fig.2b: $c=0.25, T / T_{c}=0.85, N=600$

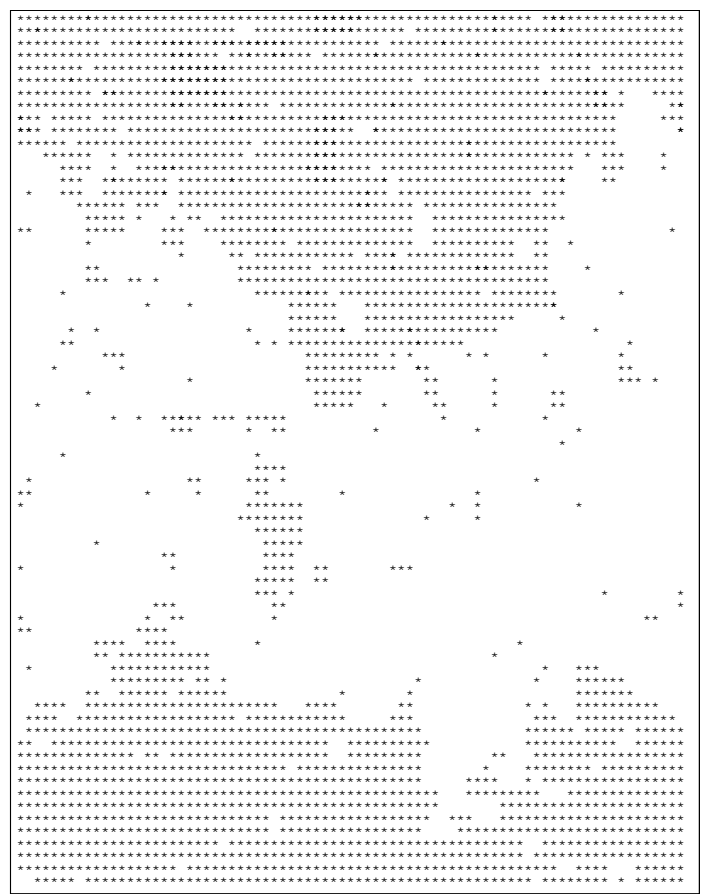

Fig.2d: $c=0.35, T / T_{c}=0.9, N=10^{7}$

Figure 2: Formation of a ghetto. 


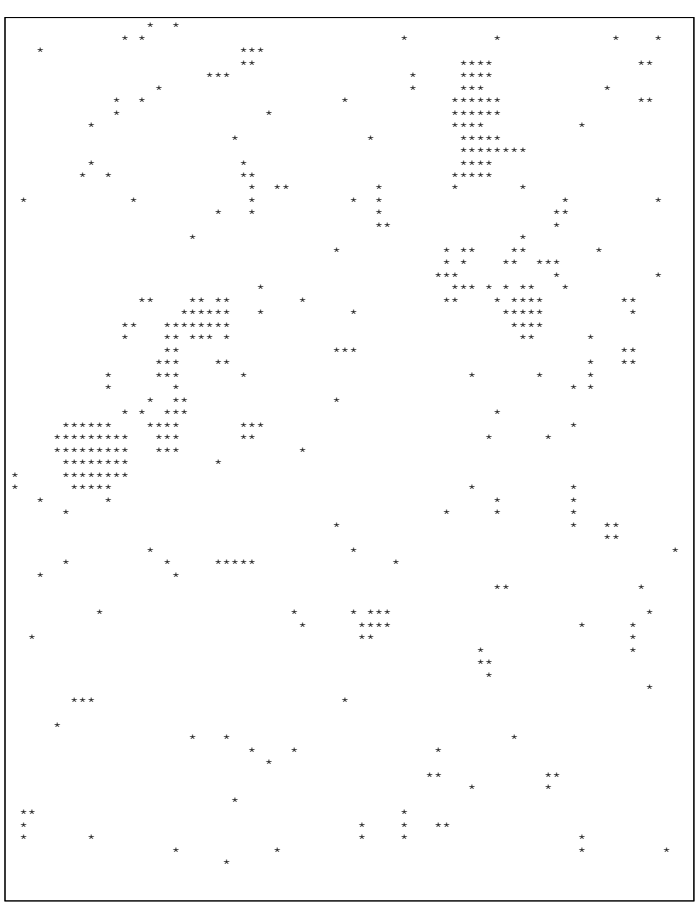

Fig.3a: $c=0.05, T / T_{c}=0.8$

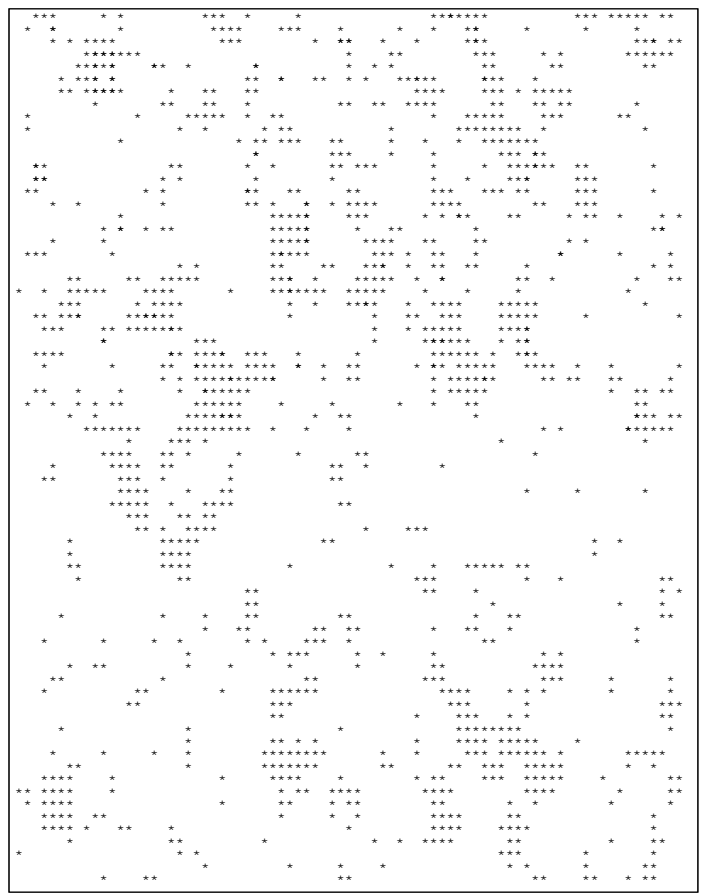

Fig.3c: $c=0.15, T / T_{c}=1.1$

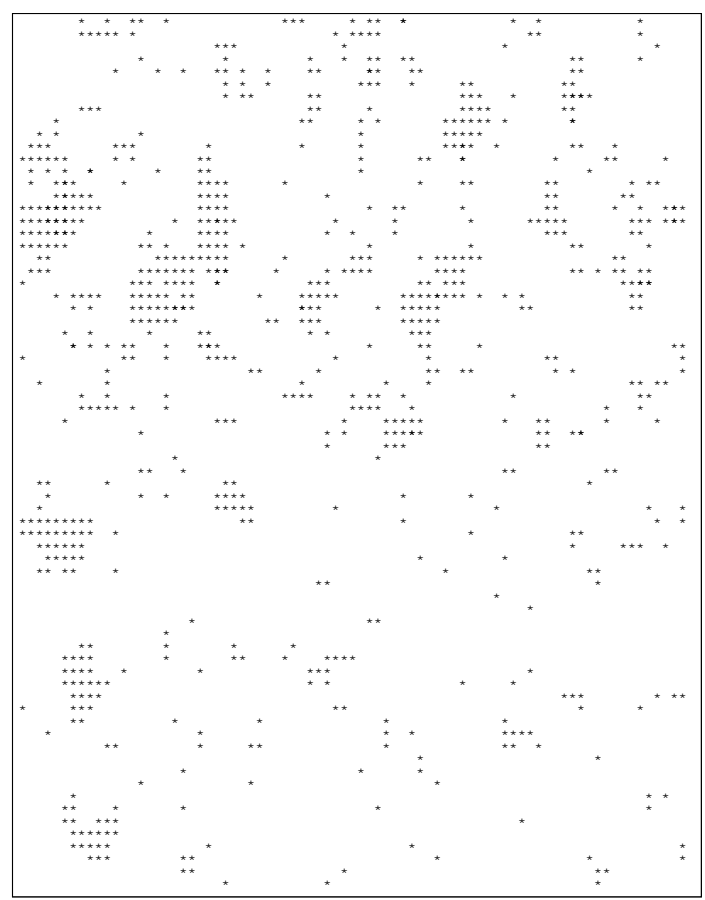

Fig.3b: $c=0.10, T / T_{c}=0.95$

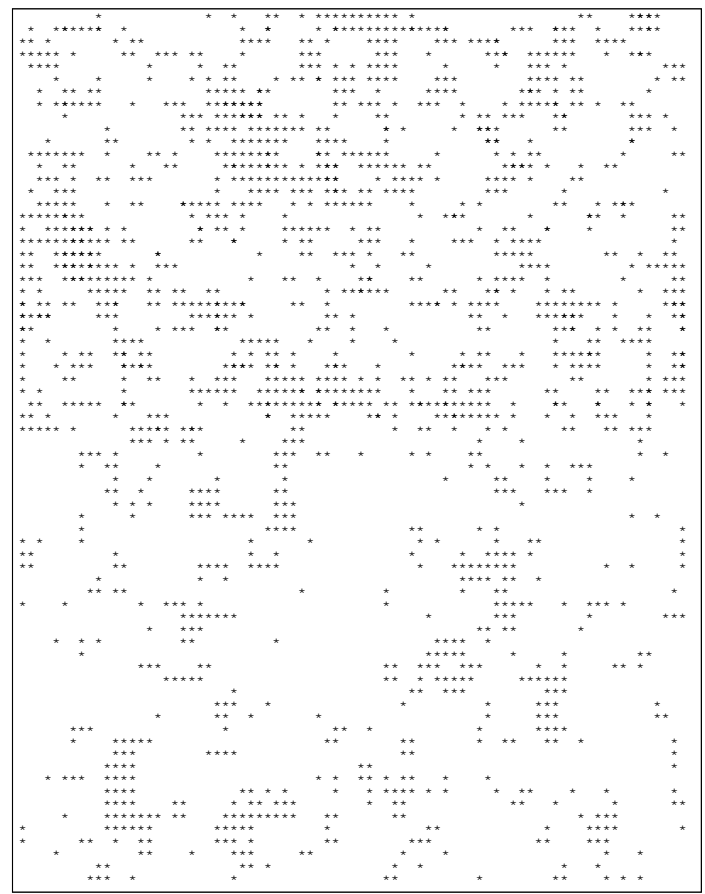

Fig.3d: $\left(c=0.2, T / T_{c}=1.25\right)$

Figure 3: No ghetto formation in equilibrium. Various concentrations and temperatures above the coexistence curve after $10^{7}$ iterations each. 


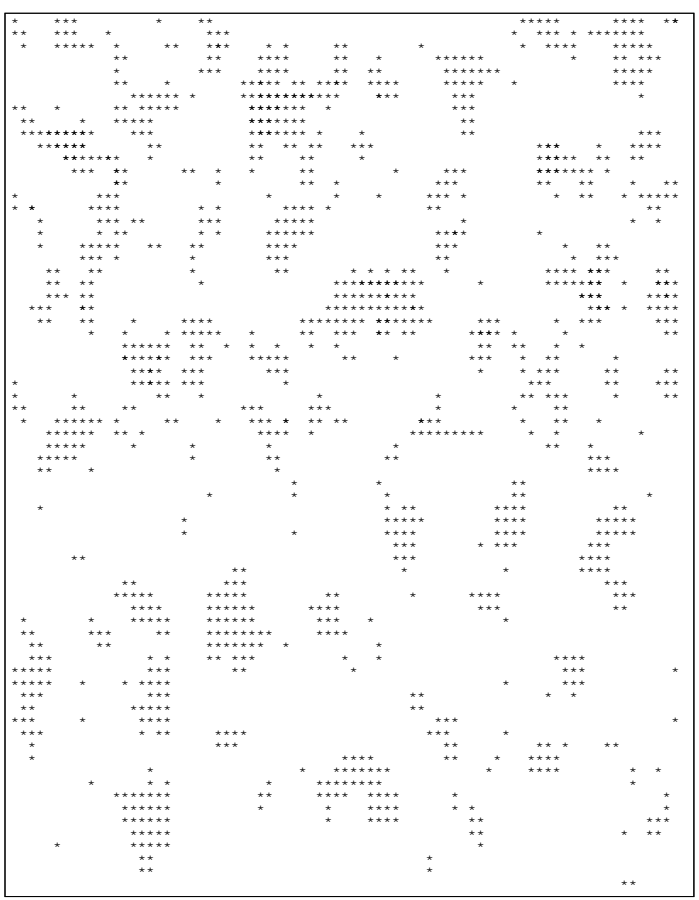

Fig.4a: $c=0.15, T / T_{c}=0.8, N=600$

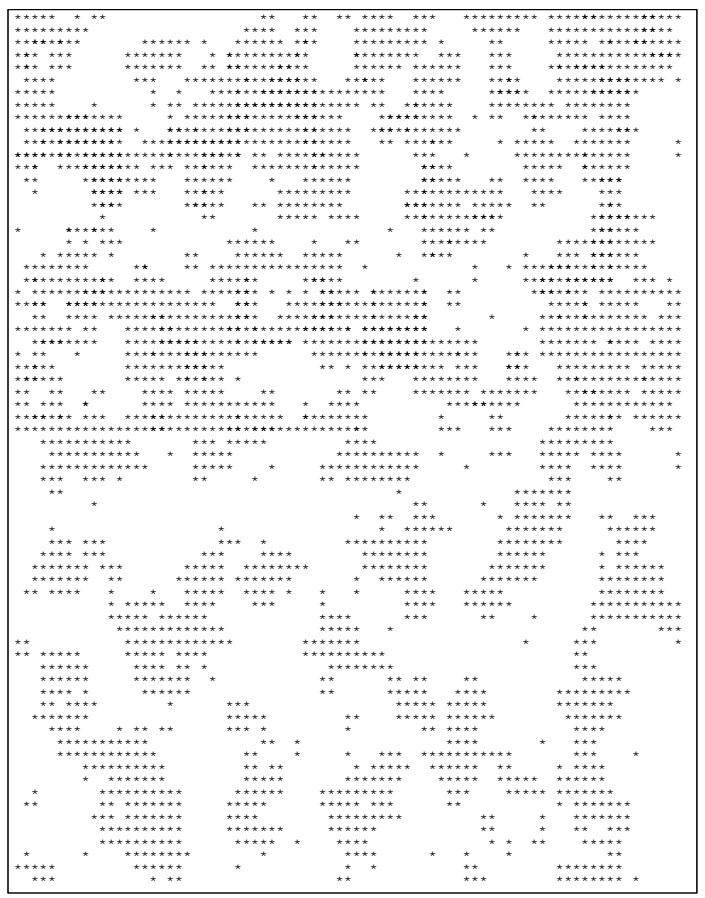

Fig.4c: $c=0.35, T / T_{c}=0.9, N=600$

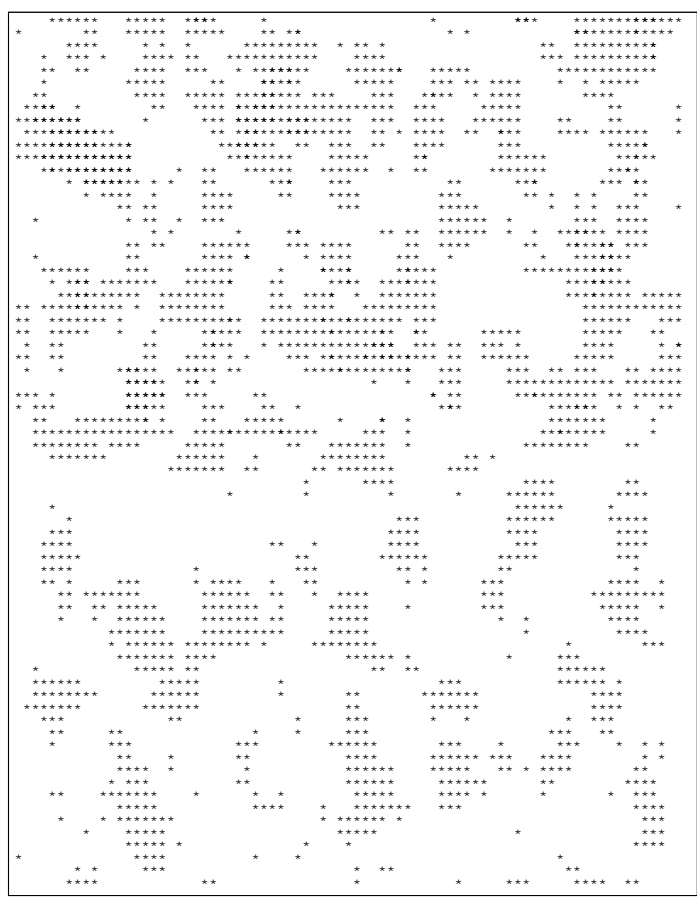

Fig.4b: $c=0.25, T / T_{c}=0.85, N=600$

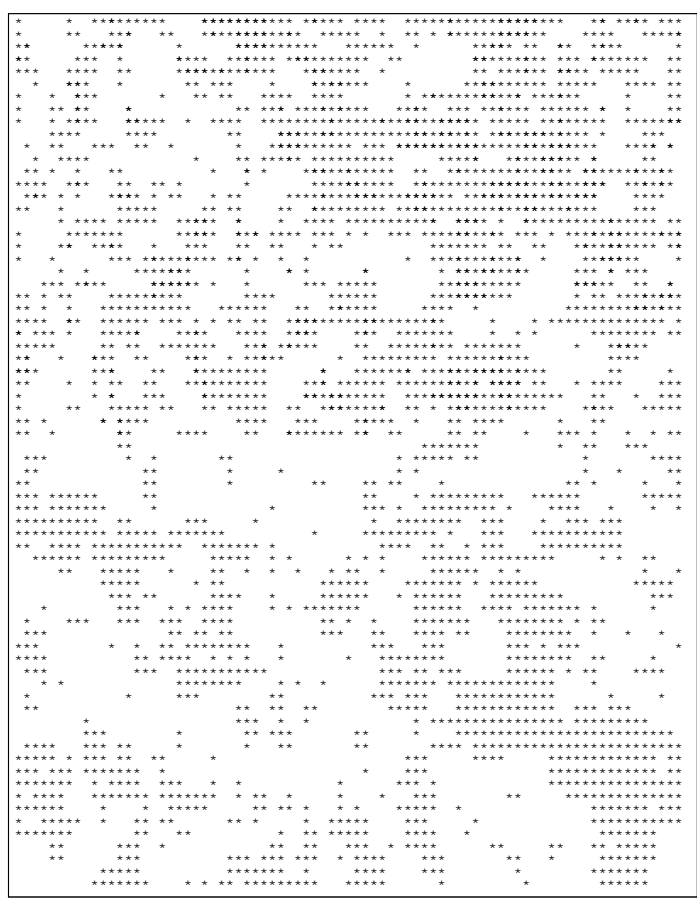

Fig.4d: $c=0.35, T / T_{c}=1.2, N=10^{7}$

Figure 4: No ghetto formation out-of equilibrium below the coexistence curve (a-c) and none above the coexistence curve (d). 